FUNDACIÓN KOINONIA (F.K). Santa Ana de Coro. Venezuela.

Manuel Ignacio Espinoza-López; Zoila Guillermina Torres-Palchisaca

http://dx.doi.org/10.35381/r.k.v6i4.1565

\title{
La Educación Física como medio de Desarrollo Motriz en Estudiantes con Discapacidad Cognitiva
}

\section{Physical Education as a Means of Motor Development in Students with Cognitive Disabilities}

\author{
Manuel Ignacio Espinoza-López \\ manuel.espinoza.83@est.ucacue.edu.ec \\ Universidad Católica de Cuenca, Azogues \\ Ecuador \\ https://orcid.org/0000-0001-6239-3653 \\ Zoila Guillermina Torres-Palchisaca \\ ztorresp@ucacue.edu.ec \\ Universidad Católica de Cuenca, Azogues \\ Ecuador \\ https://orcid.org/0000-0003-3078-6465
}

Recepción: 30 de agosto 2021 Revisado: 20 de septiembre 2021 Aprobación: 15 de noviembre 2021 Publicación: 01 de diciembre 2021 
Revista Arbitrada Interdisciplinaria KOINONIA

Año VI. Vol VI. N4. Edición Especial: Educación III. 2021

Hecho el depósito de Ley: FA2016000010

ISSN: 2542-3088

FUNDACIÓN KOINONIA (F.K). Santa Ana de Coro. Venezuela.

Manuel Ignacio Espinoza-López; Zoila Guillermina Torres-Palchisaca

\title{
RESUMEN
}

La discapacidad cognitiva ocasiona en muchos casos limitaciones psicomotoras, excluyendo a los estudiantes del sistema educativo, por lo cual se llevó a cabo este estudio donde se analiza la importancia de mejorar la atención y participación de estudiantes con este tipo de discapacidad, en especial en el área donde se requiere destrezas motrices como la Educación Física, para ello se realizó una encuesta a los docentes a cargo de la materia, luego mediante un análisis estadístico se verificó aspectos de cómo se está llevando actualmente la educación en este contexto. Los resultados muestran que hace falta más preparación y capacitaciones en el rol docente, también mejorar las habilidades motrices con programas didácticos, el rendimiento físicoemocional y la participación de los padres en su formación. Se concluyó contar con el trabajo conjunto y comprometido de padres de familia, docentes e institución que contribuyan al aprendizaje significativo del estudiante con discapacidad cognitiva.

Descriptores: Educación física; expresión corporal; incapacidad. (Palabras tomadas del Tesauro UNESCO).

\begin{abstract}
Cognitive disability causes psychomotor limitations in many cases, excluding students from the educational system, which is why this study was carried out where the importance of improving the attention and participation of students with this type of disability is analyzed, especially in the An area where motor skills such as Physical Education are required. For this, a survey was carried out to the teachers in charge of the subject, then through a statistical analysis aspects of how education is currently being carried out in this context were verified. The results show that more preparation and training are needed in the teaching role, as well as improving motor skills with didactic programs, physical-emotional performance and the participation of parents in their training. It was concluded to have the joint and committed work of parents, teachers and institutions that contribute to the significant learning of students with cognitive disabilities.
\end{abstract}

Descriptors: Physical education; movement education; disabilities. (Words taken from the UNESCO Thesaurus). 


\section{INTRODUCCIÓN}

En la etapa escolar del estudiante con discapacidad cognitiva se resalta un periodo de experiencias sobre la falta de conocimiento por parte de docentes en el desarrollo de habilidades motrices, por lo cual es necesario conocer las posibilidades de los estudiantes a través de la planificación, organización y la atención que se requiere. En el presente trabajo mostrando un recorrido teórico se va a resaltar la importancia de la educación psicomotriz en el aprendizaje del estudiante con discapacidad cognitiva en forma integrada, que puedan servir de gran interés y provecho en el mundo exigente de la educación. Igualmente permitirá desarrollar distintas habilidades y estrategias propias siguiendo las pautas evolutivas y la adquisición de rutinas motrices; a su vez la habilidad de comprender y transmitir información, de esta manera el docente adoptará las medidas de atención oportunamente, desarrollando así un aporte significativo para las instituciones educativas que promuevan a trabajar las habilidades y destrezas psicomotrices en los estudiantes que tienen alguna discapacidad.

La relación entre la incorporación al sistema escolar y la discapacidad cognitiva, viene dada por que este tipo de estudiantes presenta dificultades para el desarrollo de competencias en especial aquellas que requieren de habilidades motrices (Cadme et al. 2019), como las relacionadas a asignaturas prácticas de las ciencias, físicas, químicas y matemáticas. Anteriormente las teorías educativas, recomendaban que los niños con algún tipo de discapacidad fuesen incorporados a escuelas destinadas a la atención de estudiante con algún tipo de limitación (Manríquez, 2019), sin embargo, las nuevas teorías pedagógicas y la ampliación de los derechos sociales, recomiendan que estos niños reciban el mismo trato y sean incorporados a escuelas regulares.

No obstante, a pesar del propósito de insertar a los estudiantes con discapacidad al sistema de educación formal, muchas veces los docentes presentan deficiencia en cuanto a las estrategias de aprendizaje y el uso del material didáctico para atender a los estudiantes con algún tipo de discapacidad (Hernández, 2017), lo cual es requerido para 
poder realizar el proceso de inclusión de manera exitosa. En ese sentido se han desarrollado una infinidad de recursos didácticos, que intuyen métodos establecidos en tecnologías de la información y comunicación (TIC), recursos pedagógicos de tipo lúdico y programas informáticos que facilitan el aprendizaje de los estudiantes con algún tipo de discapacidad cognitiva, lo que ha reducido la brecha de aprendizaje entre los estudiantes con discapacidad cognitiva y los estudiantes regulares (Sánchez, Morua, Cedeño y García, 2020).

Una de las estrategias que mejoran las competencias de los estudiantes con discapacidad cognitiva en especial aquellas relacionadas a limitaciones motrices es la actividad física (Torres-Campos et al. 2021), la cual promueve habilidades y destrezas que mejoran la motricidad de los estudiantes con discapacidad cognitiva, por lo tanto, el objetivo de este artículo es valorar el uso de la educación física como estrategia para mejorar la motricidad de los estudiantes con discapacidad cognitiva.

\section{Referencial Teórico}

El concepto de discapacidad cognitiva ha sido motivo de varios debates en los últimos años, originando una serie de definiciones y con el mismo han surgido diferentes modelos teóricos. A pesar de esto es imposible explicar la discapacidad a través de un único modelo o definición (Pérez-Dalmeda \& Chhabra, 2019). Mientras que, para la OMS, la discapacidad cognitiva es definida como la incapacidad que tienen las personas para desarrollar las habilidades cognitivas durante su etapa de crecimiento, lo que lleva a que las mismas tengan dificultades para entender conceptos e instrucciones, aprender y desarrollar destrezas y habilidades e incluso memorizar, esto se manifiesta en severas limitaciones en el plano cognitivo, motor, comunicacional y social (Organización Mundial de la Salud, 1992). Dentro del proceso de la clasificación del coeficiente intelectual, el grado de severidad de la discapacidad cognitiva puede ser: 
Leve: cuando las personas cuentan con un cociente intelectual entre 50 y 70 lo que causa un retraso a nivel cognitivo y afecta directamente al campo psicomotriz, son personas capaces de alcanzar todos los grados escolares y desempeñar un trabajo regular (Aponte et al. 2017).

Moderada: cuando las personas poseen un cociente intelectual por debajo de 50 , lo que forja a contar con mayor atención y supervisión tanto en el ambiente educativo o profesional para velar que no se cometan anomalías durante el desempeño de las personas (Menjura \& Ríos, 2020).

Grave: esta condición acontece cuando el cociente intelectual se halla entre 20 y 35 , las personas con este estado deben ser examinadas firmemente, debido a sus habilidades reducidas, poca o nula compresión lectora y numérica, comunicación en base a holofrases, en el ámbito jurídico son considerados incapaces de tomar sus propias decisiones (Ke \& Liu, 2017).

Profunda: Se presenta cuando las personas poseen una capacidad intelectual por debajo de 20, lo que produce que necesariamente cuenten con las debidas atenciones de forma permanente, la supervivencia es muy reducida debido a los problemas neurológicos y la poca comunicación que tienen. (Martos-Pérez et al., 2018).

La severidad de la discapacidad cognitiva, puede estar relacionada a la etiología u origen de la discapacidad, en este sentido, entre las causas de la discapacidad cognitiva se pueden señalar las siguientes: de origen genético por la ocurrencia de alguna enfermedad como el síndrome de X frágil (Saldarriaga-Gil et al. 2020), fenilcetonuria (Sánchez-Reyna \& Quispe-Castañeda, 2020) y el síndrome de Lesch-Nyhan (CampoloGonzález et al. 2018). Otras de las causas de la discapacidad cognitiva son los trastornos cromosómicos, siendo los trastornos más frecuentes el síndrome de Down (Rachubinski 
et al., 2017); el síndrome de Prader-Willi (Carrazana \& Carrazana, 2021) y el síndrome de Angelman (Laquihuanaco et al. 2019).

Así mismo existen causas de origen biológico y orgánico, que aparecen antes, durante o después del nacimiento de una persona, entre los cuales se puede mencionar: el consumo de drogas o alcohol por la madre durante el tiempo de embarazo y después del nacimiento por traumatismos y enfermedades como la meningitis (Laquihuanaco et al. 2019), las cuales ocasionan: falta o retraso en el desarrollo de habilidades motoras, destrezas, lenguaje y habilidades de autoayuda, produciendo un déficit en el crecimiento adecuado, problemas en el comportamiento, imposibilidad para adaptarse o ajustarse a nuevas situaciones y dificultad para entender y acatar las diferentes normas sociales.

Por otra parte, la falta de desarrollo psicomotriz en estudiantes con discapacidad cognitiva provoca problemas específicos de adquisición de habilidades motrices básicas, del control de sus funciones corporales y la falta de equilibrio, entre otras (CamposGonzález et al. 2021), no obstante el desarrollo motor de cada individuo con discapacidad cognitiva varía dependiendo de factores como el nivel cognitivo, ya que personas con una discapacidad leve pueden llegar a tener un desarrollo normal y eficaz en su motricidad; en cambio aquellas con una deficiencia cognitiva severa pueden presentar un menor desarrollo motriz (Alonso, 2018), caracterizado por deficiencias a nivel de percepción, atención, memoria y recreación de pensamientos (ValarezoMendoza et al. 2017).

Adicionalmente las personas con discapacidad cognitiva se caracterizan por poseer hipermovilidad articular y, como consecuencia de ella, se genera un alto grado de movimiento de sus articulaciones, como en caso de la rótula y la poca estabilización de la cabeza a nivel cervical, lo cual lleva a problemas motrices para la realización de actividades de índole practico (Carbonell-Bobadilla et al. 2020).

Dado lo expresado en los párrafos previos en relación a las peculiaridades de motricidad de las personas con discapacidad cognitivas, los niños con este tipo de condición suelen 
Revista Arbitrada Interdisciplinaria KOINONIA

Año VI. Vol VI. N4. Edición Especial: Educación III. 2021

Hecho el depósito de Ley: FA2016000010 ISSN: 2542-3088

FUNDACIÓN KOINONIA (F.K). Santa Ana de Coro. Venezuela.

Manuel Ignacio Espinoza-López; Zoila Guillermina Torres-Palchisaca

tener dificultades en la coordinación, y llegando a ser torpes, o tener movimientos excesivos, obteniendo situaciones críticas, cuando la discapacidad cognitiva es grave, donde pueden presentarse movimientos sin finalidad, e incluso en casos más severos se pueden presentar conductas destructivas, agresivas y violentas (Ke \& Liu, 2017).

\section{La Educación Física como medio para el desarrollo motriz en niños con discapacidad cognitiva}

Para lograr el progreso de las habilidades motrices es necesario establecer una estrecha relación entre las actividades físicas recreativas que actualmente se da en las clases de Educación Física y el poco desarrollo motriz logrado por los estudiantes, sin embargo este proceso se ve limitado por la poca praxis pedagógica que ejecutan los docentes, en la aplicación de esta herramienta pedagógica, por lo tanto es necesario establecer mejor el desarrollo de las actividades físicas recreativas, que deben ser adecuadas y encaminadas a fortalecer las capacidades y su desarrollo motriz, también incentivar la participación dirigido hacia la inclusión social (Torres et al. 2017). Por otro lado la Educación Física cuenta con la propuesta de Actividad Física Adaptada (AFA), orientado a identificar y solucionar problemas psicomotores frente a la discapacidad y a través de esto poder insertarse en el proceso educativo de forma normal, mediante actividades como la danza, deportes acuáticos a lo largo de su vida, que apoyen la integración y la inclusión, para ello es necesario contar con programas e infraestructura apropiadas sin olvidar el seguimiento individual de cada participante (Muñoz et al., 2017).

Además, en una experiencia llevada a cabo con niños diagnosticados con discapacidad intelectual, se diseñó de un programa de ejercicios físicos con el objetivo de incidir en el desarrollo psicomotriz de los niños. Obteniendo como resultado que el programa conformado por Gimnasia Básica, Actividades Rítmicas y Juegos, era capaz de estimular el desarrollo psicomotriz, incidiendo en el incremento en las habilidades motoras básicas y las capacidades físicas (Espinosa-Telles, 2011). 
En este contexto la Educación Física inclusiva ampara a toda la población estudiantil de acuerdo al origen de las Necesidades Educativas Especiales (NEE), que se puedan presentar, promoviendo la participación conjunta mediante diversas estrategias metodológicas, respetando diferencias individuales y fortaleciendo los contenidos curriculares para adquirir el aprendizaje deseado, tomando en cuenta como punto de partida sus capacidades actuales en el aspecto motriz y cognitivo. En esta línea entre las metodologías inclusivas que pueden atender a las diferentes necesidades está el trabajo cooperativo que consiste en formar pequeños grupos que puedan responder a las particularidades de cada estudiante, logrando una mejor interacción y que el estudiante se comprometa aún más con su propio aprendizaje y el de sus compañeros (Monge \& Monge, 2009).

Igualmente es necesario, agregar en las planificaciones de cada institución respectivamente actividades que permitan al estudiante con discapacidad cognitiva formar parte de una clase normal y no ser excluido por su discapacidad, para lo cual es preciso contar con la colaboración activa de los padres de familia, para organizar actividades deportivas y recreativas, por otro lado motivar al estudiante con discapacidad cognitiva a practicar diversas disciplinas que requieran actividades físicas para identificar sus capacidades y preferencias, así se enriquecer más su autoestima y su emoción por participar en distintas actividades.

\section{MÉTODO}

El diseño del presente estudio corresponde a un enfoque de investigación cuantitativo, de tipo descriptiva con diseño no experimental. Para llevar a cabo la presente investigación la población estuvo conformada por los docentes que dictan clases de Educación Física dentro de la provincia del Cañar, se aplicó un muestreo probabilístico por conglomerado a 70 docentes. 
Para el desarrollo de recolección de datos de la presente investigación se generó una encuesta vía online mediante Google forms, aplicándose 10 preguntas las mismas que fueron sometidas a validación por juicio de expertos en el área y como instrumento de recolección de datos de la encuesta, se utilizó el tratamiento estadístico mediante el SPSS con estadísticos descriptivos de porcentajes y frecuencias.

\section{RESULTADOS}

Tabla 1.

Resultados de la encuesta realizada a los docentes de Educación Física sobre: La Educación Física como medio de desarrollo motriz en estudiantes con discapacidad cognitiva.

1. ¿Cuál es su edad?

\begin{tabular}{ccc}
\hline Promedio de Edades & \multicolumn{2}{c}{ N0 } \\
\hline 36,26 & & \\
\hline 2. ¿Cuántos años lleva en la docencia? & Frecuencia & Porcentaje \\
\hline de 1 a 5 años & 24 & 34,3 \\
de 5 a 10 años & 17 & 24,3 \\
de 10 a 15 años & 10 & 14,3 \\
más de 15 años & 19 & 27,1 \\
Total & 70 & 100,0 \\
\hline
\end{tabular}

3. ¿Con qué frecuencia ha podido trabajar el desarrollo motriz de los estudiantes con discapacidad cognitiva?

\begin{tabular}{lcc}
\hline Siempre & 16 & 22,9 \\
Casi Siempre & 17 & 24,3 \\
Ocasionalmente & 26 & 37,1 \\
Casi Nunca & 10 & 14,3 \\
Nunca & 1 & 1,4 \\
Total & 70 & 100,0 \\
\hline
\end{tabular}

4. ¿Con qué frecuencia la Educación Física puede ayudar a mejorar la motricidad en estudiantes con discapacidad cognitiva? 
Revista Arbitrada Interdisciplinaria KOINONIA

Año VI. Vol VI. N4. Edición Especial: Educación III. 2021

Hecho el depósito de Ley: FA2016000010 ISSN: 2542-3088

FUNDACIÓN KOINONIA (F.K). Santa Ana de Coro. Venezuela.

Manuel Ignacio Espinoza-López; Zoila Guillermina Torres-Palchisaca

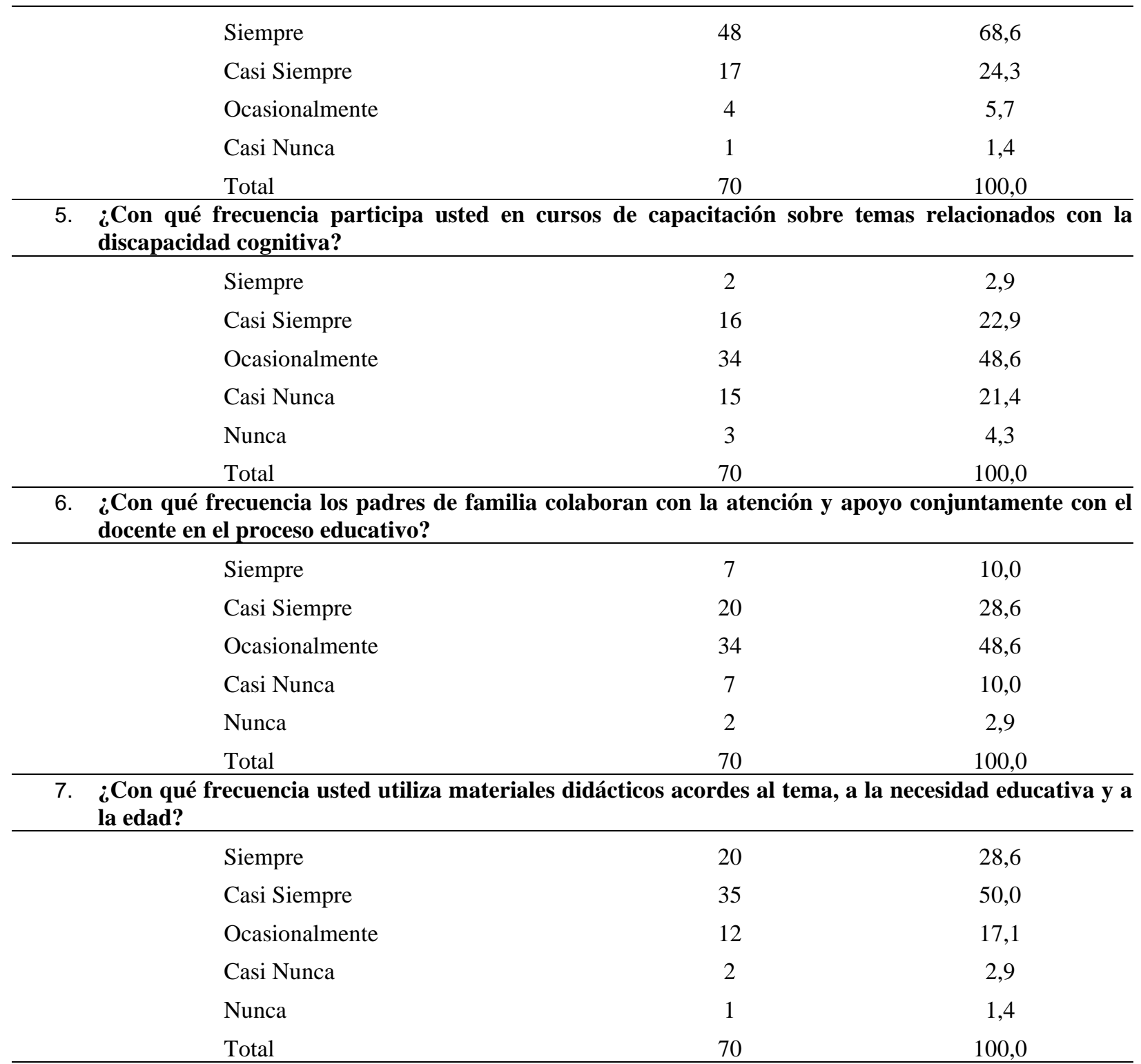

8. ¿Con qué frecuencia realizan reuniones los docentes del área de Educación Física y de aula para buscar o seleccionar estrategias, nuevos métodos a aplicar y analizar el desarrollo motriz de estudiantes con discapacidad cognitiva? 


\begin{tabular}{ccc}
\hline Siempre & 13 & 18,6 \\
Casi Siempre & 22 & 31,4 \\
Ocasionalmente & 20 & 28,6 \\
Casi Nunca & 14 & 20,0 \\
Nunca & 1 & 1,4 \\
Total & 70 & 100,0 \\
\hline 9. ¿En qué medida sería necesario integrar actividades físicas recreativas, programas de ejercicios físicos, \\
actividades relacionadas con la danza y deportes acuáticos mediante estrategias especializadas \\
propiamente para el desarrollo motriz en estudiante? & \\
\hline Siempre & 41 & 58,6 \\
Casi Siempre & 20 & 28,6 \\
Ocasionalmente & 6 & 8,6 \\
Casi Nunca & 2 & 2,9 \\
Nunca & 1 & 1,4 \\
Total & 70 & 100,0 \\
\hline 10. ¿Cuál es su nivel de conocimiento acerca de los procesos de una planificación con adaptaciones \\
curriculares grado 3, para estudiantes con discapacidad cognitiva? \\
\hline Muy Alto & 1 & 1,4 \\
Alto & 14 & 20,0 \\
Medianamente & 35 & 50,0 \\
Poco & 19 & 27,1 \\
Nada & 1 & 1,4 \\
Total & 70 & 100,0 \\
\hline
\end{tabular}

Fuente: Elaboración Propia

\section{DISCUSION}

Los resultados de la encuesta realizada a los docentes de Educación Física sobre: "La Educación Física como medio de desarrollo motriz en estudiantes con discapacidad cognitiva", reflejan que la edad promedio de los 70 docentes consultados estuvo entre los 36,26 años. Al total de encuestados se les consulto cuanto tiempo llevaban en la docencia donde el mayor grupo estuvo ubicado entre 1 a 5 años de experiencia con un porcentaje de 34,3\%, mientras que el menor número de docentes reporto tener entre 10 a 15 años en la docencia (14,3\%) (pregunta 2). 
En cuanto a la frecuencia con la que han podido trabajar el desarrollo motriz de los estudiantes con discapacidad cognitiva, se reportó que la mayoría de docentes respondió la opción ocasionalmente con un promedio de 37,1\%, y además consideran en su mayoría (68,6\%) que la Educación Física ayuda a mejorar la motricidad en estos estudiantes, por lo tanto, se habla sobre la formación adecuada, la predisposición del profesor, la evaluación constante que se realiza sobre la Educación Física, la unión y el uso de recursos necesarios para que se lleve a cabo. El practicar la Educación Física frecuentemente resulta una opción importantísima para el desarrollo de los trastornos que puedan presentar los niños con cierta discapacidad intelectual, siendo un espacio adecuado para el apoyo de todas las actividades dirigidas a la corrección o ajuste de los defectos que se deriven de alteraciones en el sistema nervioso (Gómez-Valdés et al. 2019).

En relación a la quinta pregunta sobre la frecuencia de participación en cursos de capacitación sobre temas relacionados con la discapacidad cognitiva, las respuestas en su mayoría fueron ocasionalmente con un $48,6 \%$ lo que significa que la mayoría de docentes carece de preparación adecuada para trabajar con esta población de estudiantes que a pesar de ser una minoría dentro de los programas regulares de educación, se merecen la mejor atención al ser un grupo vulnerable y para lograr una correcta inserción al sistema educativo es necesario que el docente cuente con las herramientas adecuadas y las habilidades requeridas para proveer un acompañamiento de soporte al niño.

A su vez se puede contribuir brindando soluciones y así proveer mejor atención a los estudiantes, evitando las discriminaciones por las diferentes patologías y ofreciendo un profesional que se caracterice por brindar educación de calidad a los niños. Por lo anteriormente mencionado, se puede decir que es responsabilidad primeramente del Estado, y en segunda instancia del plantel proveer a los docentes la posibilidad de capacitarse de forma continua para que puedan brindar una enseñanza con eficacia y 
conforme con las necesidades de los niños con condiciones especiales (López-Jiménez \& Noguera-Coronado, 2019).

Por otro lado, los padres de familia de los niños con discapacidad cognitiva, son los primeros llamados a ser el soporte dentro del proceso de enseñanza aprendizaje, sin embargo, se ha podido observar que ocasionalmente el $48,6 \%$ están presentes y apenas un $10 \%$ lo hace siempre, lo que lleva a pensar que lamentablemente existe despreocupación del primer núcleo de la sociedad, para mejorar la calidad de vida de estos niños.

Al respecto, (Armero-Urquiza et al. 2019), plantean una propuesta para que los padres de estudiantes con discapacidad intelectual puedan intervenir activamente en el desarrollo de la educación de sus hijos de forma optimista y que comprendan la importancia de los ejercicios físicos y de la estimulación en la corrección de este tipo de discapacidades y entorno a ello puedan brindar el apoyo que se requiere.

Con respecto a la frecuencia con que los docentes usan materiales didácticos acordes al tema, a la necesidad educativa y a la edad, las opciones con mayor y menor porcentaje fueron casi siempre con un $50 \%$ y nunca con 1,4\% respectivamente. Es necesario puntualizar que la utilización de materiales didácticos acordes con el tema, y a las necesidades que presenten los alumnos con discapacidad física o motora, se encuentren siempre a las posibilidades de ejecución, acción y movimiento, pues el docente deberá tener conocimiento de la variedad de recursos didácticos y como emplearlos dependiendo del grado y tipo de discapacidad, para lo cual es necesario que la toma de decisiones sean oportunas para el caso, también se debe tener en cuenta que este tipo de personas van a estar limitadas en situaciones cuando se involucre actividades con el uso del material didáctico (Sandoval-Mena et al. 2019).

No cabe duda que el trabajo interdisciplinario es fundamental dentro de todo proceso educativo y el análisis de las diferentes problemáticas que se presenten durante este proceso entre compañeros de un área específica resulta muy enriquecedor, por esto es 
muy necesario que se dé continuamente esta sinergia, sin embargo en lo referente a este cuestionamiento, los docentes encuestados, apenas el 31,4\% busca casi siempre el apoyo en sus compañeros de área para buscar estrategias que mejoren la participación en clases de estudiantes con discapacidad intelectual. Por tal razón, así como es necesario que el docente sea capacitado también es necesario que sea multiplicador del conocimiento impartiendo enseñanzas y buscando soluciones a diferentes situaciones que se puedan presentar en las clases (Armero-Urquiza et al. 2019).

En relación al cuestionamiento sobre en qué medida sería necesario integrar actividades físicas recreativas, programas de ejercicios físicos, actividades relacionadas con la danza y deportes acuáticos mediante estrategias especializadas propiamente para el desarrollo motriz en el estudiante con discapacidad, el 58,6 \% demostraron su aceptación a incluir estas actividades en el programa de trabajo ya que la estimulación temprana, por medio del deporte adaptado a las limitaciones motoras en niños de edades entre 5 y 6 años que presentan cierta discapacidad mental, dan como resultado un correcto desarrollo e inserción social (Sandoval-Mena et al. 2019).

Con respecto a la adaptación con grado 3 para estudiantes con discapacidad cognitiva, Escudero (2017), señala que, el docente tiene que tener una preparación efectiva donde pueda dominar y desarrollar adecuadamente una planificación con adaptación curricular dependiendo del tipo de discapacidad y el grado que se debe aplicar, en tal razón en el cuestionamiento hecho a los docentes el $50,0 \%$ siendo la mitad, manifiestan que medianamente conocen sobre este proceso, con ello se comprueba que en el sistema educativo es poco aplicado, por la falta de capacitaciones, la preparación necesaria, el seguimiento requerido y por ello las adaptaciones resultan en varios casos poco significativas y nada eficientes. Es importante que todos tengan conocimiento pleno para saber atender a las necesidades educativas especiales que con frecuencia se presenta en las instituciones educativas. 
Entorno a esto se afirma que las adaptaciones curriculares son el instrumento esencial para lograr incluir individualmente al estudiante con NEE, en el ámbito educativo, y pueda trabajar de acuerdo a sus posibilidades, es por ello que se realizan los ajustes necesarios en la programación curricular para entender las diferencias individuales del alumnado y emprender actividades conformes a su desarrollo. Siendo estas las medidas de flexibilización del currículo escolar, orientadas a que los estudiantes accedan a una enseñanza personalizada y obtengan promedios y beneficios apropiados.

\section{CONCLUSIONES}

La participación optima de los estudiantes con discapacidad cognitiva en el área de Educación Física y que esto favorezca su desarrollo motriz, ha sido el efecto primordial de este estudio donde se pudo analizar experiencias y perspectivas que los docentes tienen actualmente sobre este proceso.

Asegurar un aprendizaje equilibrado y enriquecedor a todos los estudiantes sin discriminación y distinción por sus condiciones de discapacidad, es lo que se debe ofrecer en las instituciones educativas, priorizando los aprendizajes que motiven al estudiante a participar con emoción y autoestima, al mismo tiempo que sean adecuados de acuerdo a sus características, trabajar en la efectividad y eficacia de los procesos de enseñanza y aprendizaje ajustando y aplicando métodos y estrategias necesariamente para alcanzar un rendimiento mejor, ya que se ha podido verificar que el estudiante con discapacidad cognitiva va a necesitar siempre una atención especializada.

Por tal razón para mejorar la atención de los estudiantes con discapacidad cognitiva se deben emprender acciones: en primer lugar; los docentes deben priorizar sus capacitaciones en el desarrollo de adaptaciones curriculares y en el uso de recursos didácticos en particular cuando la discapacidad afecta las destrezas motrices, esta puede incluir el uso de recursos lúdicos para motivar la participación, en segundo lugar se debe hacer una campaña de concientización para incorporar a aquellos padres que sean 
involucrados en el proceso educativo de sus hijos con discapacidad cognitiva y por último se deben clasificar y aplicar actividades físicas, deportivas y recreativas que ayuden a mejorar el desarrollo motriz en estudiantes con discapacidad cognitiva, conjuntamente con los coordinadores del área.

Para que este proceso sea efectivo en las clases de Educación Física y los estudiantes con discapacidad cognitiva logren un buen desarrollo motriz, resulta importantísimo que exista una colaboración permanente entre docentes, padres de familia e institución pues solo el trabajo conjunto y comprometido de todos estos actores podrá contribuir a la adquisición de un aprendizaje significativo e integral del estudiante con NEE.

\section{FINANCIAMIENTO}

No monetario.

\section{AGRADECIMIENTO}

A la Universidad Católica de Cuenca; por todo el apoyo brindado en la motivación y desarrollo de esta investigación.

\section{REFERENCIAS CONSULTADAS}

Alonso, D. (2018). Desarrollo de las habilidades motrices de las personas con discapacidad intelectual a través del proceso cognitivo [Development of the motor skills of people with intellectual disabilities through the cognitive process]. Artseduca, (19), 224-245. revistes.uji.es/index.php/artseduca/article/view/2789

Aponte, A., Carvajal, S. R., González, L. D., \& Fandiño, A. M. (2017). Relaciones de equivalencia en niños con discapacidad cognitiva leve o inteligencia limítrofe [Equivalence relations in children with mild cognitive disability or borderline intelligence]. Revista Evaluación e Intervención Psicológica, 2(1), 1-11. http://190.65.221.162:1094/Revistas/index.php/UNINCCA/article/viewFile/59/73 
Revista Arbitrada Interdisciplinaria KOINONIA

Año VI. Vol VI. N4. Edición Especial: Educación III. 2021

Hecho el depósito de Ley: FA2016000010 ISSN: 2542-3088

FUNDACIÓN KOINONIA (F.K). Santa Ana de Coro. Venezuela.

Manuel Ignacio Espinoza-López; Zoila Guillermina Torres-Palchisaca

Armero-Urquiza, I. D. L. A., Alfonso Nazco, M., \& Martell Cuellar, D. (2019). Folleto para la orientación a familias con menores de 7-9 años que presentan discapacidad intelectual leve [Brochure for guidance to families with children under 7-9 years old who have mild intellectual disability]. Atlante Cuadernos de Educación y Desarrollo, (junio). https://n9.cl/f1z3z

Cadme, M. L.; Casas, X. C., \& Loor, S. B. (2019). Alteraciones psicomotrices y la relación en el desempeño ocupacional educativo [Psychomotor alterations and the relationship in educational occupational performance]. Cuidado y Ocupación Humana, (8), 45-52. https://n9.cl/0d2vm

Campolo-González, A., Vargas Díaz, A., Fontboté Riesco, D., \& Hernández Chávez, M. (2018). Síndrome de Lesch-Nyhan y automutilación oral [Lesch-Nyhan syndrome and oral self-mutilation]. Revista chilena de pediatría, (89), 86-91. http://dx.doi.org/10.4067/S0370-41062018000100086

Carbonell-Bobadilla, N., Rodríguez-Álvarez, AA, Rojas-García, G, Barragán-Garfias, JA, Orrantia-Vertiz, M. \& Rodríguez-Romo, R. (2020). Síndrome de hipermovilidad articular [Joint hypermobility syndrome]. Acta Ortopédica Mexicana, (34), 441-449. https://dx.doi.org/10.35366/99145

Carrazana, C. E. C. \& Carrazana, C. G. (2021). Síndrome de Prader-Willi. A propósito de un caso [Prader-Willi syndrome. About a case]. Revista Finlay, (11). http://revfinlay.sld.cu/index.php/finlay/article/view/869

Escudero, N. (2017). Análisis de la propuesta de adaptaciones curriculares generadas en el currículo educativo ecuatoriano 2016 [Analysis of the proposal of curricular adaptations generated in the Ecuadorian educational curriculum 2016]. Machala: Universidad Técnica de Machala, http://repositorio.utmachala.edu.ec/handle/48000/9979

Espinosa-Telles, Y. (2011). Programa de ejercicios físicos para el desarrollo psicomotriz de niños con discapacidad intelectual [Physical exercise program for the psychomotor development of children with intellectual disabilities]. Efdeportes. com, revista digital, (160). https://n9.cl/hjpkd 
Revista Arbitrada Interdisciplinaria KOINONIA

Año VI. Vol VI. N4. Edición Especial: Educación III. 2021

Hecho el depósito de Ley: FA2016000010 ISSN: 2542-3088

FUNDACIÓN KOINONIA (F.K). Santa Ana de Coro. Venezuela.

Manuel Ignacio Espinoza-López; Zoila Guillermina Torres-Palchisaca

Gómez-Valdés, A, Planes Rivera, D, \& Gómez Ledesma, Y. (2019). Acciones metodológicas para contribuir al proceso de Educación Física Inclusiva: una aproximación al tema [Methodological actions to contribute to the process of Inclusive Physical Education: An approach to the topic]. Mendive. Revista de Educación, 17(1), 84-96.

Hernández, M. R. (2017). 565 juegos y tareas de iniciación deportiva adaptada a las personas con discapacidad [565 sports initiation games and tasks adapted to people with disabilities]. Paidotribo, https://n9.cl/x7192

Ke, X. \& Liu, J. (2017). Discapacidad intelectual [intellectual disability]. Manual de Salud Mental Infantil y Adolescente de la IACAPAP, '1-28. Recuperado desde https://n9.cl/8on3

Laquihuanaco, F., Rojas, C. \& Laquihuanaco, R. (2019). Evaluación clínica y tratamiento estomatológico de un caso con aparente síndrome de angelman [Clinical evaluation and stomatological treatment of a case with apparent angelman syndrome]. Evidencias en Odontología Clínica, (2), 53-58. http://dx.doi.org/10.35306/eoc.v2i2.707

López-Jiménez, C. C., \& Noguera-Coronado, T. J. (2019). Formación docente en inclusión de niños con necesidades educativas especiales [Teacher training in the inclusion of children with special educational needs]. (Doctoral dissertation, Universidad de la Costa). https://n9.cl/g9n53

Manríquez, R. E. (2019). Perspectiva global de una educación inclusiva, en Chile y en los principales países sudamericanos [Global perspective of an inclusive education, in Chile and in the main South American countries]. Educación, (25), 33-42. https://doi.org/10.33539/educacion.2019.v25n1.1765

Martos-Pérez, J.; Freire-Prudencio, S.; Llorente-Comí, M.; Ayuda-Pascual, R.; GonzálezNavarro, A. (2018). Autismo y cociente intelectual: ¿estabilidad [Autism and IQ: stability]. Rev. Neurol, (66), S39-44. https://n9.cl/igvf5

Menjura, M. I. \& Ríos, M. C. P. (2020). Habilidades adaptativas en niños y niñas con discapacidad cognitiva [Adaptive skills in children with cognitive disabilities]. Tempus Psicológico, https://doi.org/10.30554/tempuspsi.3.1.3621.2020

(3),

157-181. 
Revista Arbitrada Interdisciplinaria KOINONIA

Año VI. Vol VI. N4. Edición Especial: Educación III. 2021

Hecho el depósito de Ley: FA2016000010

ISSN: 2542-3088

FUNDACIÓN KOINONIA (F.K). Santa Ana de Coro. Venezuela.

Manuel Ignacio Espinoza-López; Zoila Guillermina Torres-Palchisaca

Monge, M. \& Monge, M. (2009). Cuerpos, mentes y aprendizajes diversos: la clase de Educación Física como modelo de una educación inclusiva en Costa Rica [Bodies, minds and diverse learning: the Physical Education class as a model of an inclusive education in Costa Rica]. Agora para la educación física y el deporte, 9, (Ejemplar dedicado a: Educación física y discapacidad), p. 115-136. https://n9.cl/6gnu0

Muñoz, J. Garrote, D. \& Sánchez, C. (2017). La práctica deportiva en personas con discapacidad: motivación personal, inclusión y salud [Sports practice in people with disabilities: personal motivation, inclusion and health]. International Journal of Developmental and Educational Psychology, (4), 145-151. https://www.redalyc.org/pdf/3498/349853537015.pdf

OMS (1992). ICD-10 Guide for Mental Retardation. Geneva: Division of Mental Health and Prevention of Substance Abuse, WHO. https://www.who.int/mental health/media/en/69.pdf

Pérez-Dalmeda, M. E., \& Chhabra, G. (2019). Modelos teóricos de discapacidad: un seguimiento del desarrollo histórico del concepto de discapacidad en las últimas cinco décadas [Theoretical models of disability: tracing the historical development of disability concept in last five decades]. Revista Española de Discapacidad (REDIS), (7), '7-27. https://doi.org/10.5569/2340-5104.07.01.01

Rachubinski, A., Hepburn, S., Elias, E., Gardiner, K., \& Shaikh, T. (2017). Autismo y syndrome de down: ajustar el diagnóstico y profundizar en la genética [Autism and down syndrome: adjust the diagnosis and delve into genetics]. Revista de síndrome de Down, (34), 12-17. https://n9.cl/mjc73

Saldarriaga-Gil, W., Tascon Ospina, E. ,\& Herrera-Castañeda, E. (2020). Síndrome del $X$ frágil en fecundación in vitro. Reporte de caso [Fragile $X$ syndrome in in vitro fertilization. A case report]. Revista chilena de obstetricia y ginecología, (85), 654661. http://dx.doi.org/10.4067/S0717-75262020000600654

Sánchez, B. H.; Morua, G. V.; Cedeño, G. G.; García, J. C. S. (2020). Discapacidad intelectual y el uso de las tecnologías de la información y comunicación: revisión sistemática [Intellectual disability and the use of information and communication technologies: systematic review]. Revista INFAD de Psicología. International Journal of Developmental and Educational Psychology, (2), 177-188. https://doi.org/10.17060/ijodaep.2020.n1.v2.1830 
Revista Arbitrada Interdisciplinaria KOINONIA

Año VI. Vol VI. N4. Edición Especial: Educación III. 2021

Hecho el depósito de Ley: FA2016000010 ISSN: 2542-3088

FUNDACIÓN KOINONIA (F.K). Santa Ana de Coro. Venezuela.

Manuel Ignacio Espinoza-López; Zoila Guillermina Torres-Palchisaca

Sánchez-Reyna, V. \& Quispe-Castañeda, C. (2020). Fenilcetonuria e importancia del tamiz neonatal [Phenylketonuria and importance of neonatal screening]. Revista Médica de Trujillo, (15), 185-189. http://dx.doi.org/10.17268/rmt.2020.v15i04.09

Sandoval-Mena, M., Simón Rueda, C., \& Márquez Vázquez, C. (2019). ¿Aulas inclusivas o excluyentes?: barreras para el aprendizaje y la participación en contextos universitarios [Inclusive or exclusive classrooms?: barriers to learning and participation in university contexts]. Revista Complutense de Educación, 30(1), 261-276. https://doi.org/10.5209/RCED.57266

Torres, Á., Torres, M., Guevara, P., Caraballo, G., \& Morales Neira, D. (2017). Actividades físico-recreativas y motricidad: proyecto para la inclusión de niños con discapacidad [Physical-recreational activities and motor skills: project for the inclusion of children with disabilities]. Lecturas: educación física y deportes. 22. 113. https://n9.cl/sbn7c

Torres-Campos, E., Ortiz Guadalupe, L. E., Carmenate Figueredo, Y. O., \& Toledo Sánchez, M. (2021). Estimulación motriz en niños con discapacidad intelectual [Motor stimulation in children with intellectual disabilities]. Universidad y Sociedad, (13), 378-388. https://rus.ucf.edu.cu/index.php/rus/article/view/2177

Valarezo-Mendoza, E, Bayas Cano, A, Aguilar Chasipanta, W, Paredes Navarrete, L, Paucar Ipiales, E, \& Romero Frómeta, E. (2017). Programa de actividades físicorecreativas para desarrollar habilidades motrices en personas con discapacidad intelectual [A physical recreation program to develop motor skills in people with intellectual disability]. Revista Cubana de Investigaciones Biomédicas, (36), 1-13. 